Name: Peer Review Information for "Vibrational Modes Promoting Exciton Relaxation in the B850 Band of LH2"

First Round of Reviewer Comments

Reviewer: 1

\title{
Comments to the Author
}

The authors carried out a comprehensive experimental study of the exciton relaxation in the B850 band of LH2 using two-dimensional electronic spectroscopy. By analyzing the spectra, the authors identified several vibrational modes coupled to the electronic transitions that can play an important role in the exciton relaxation, especially the modes that breaks the symmetry of the ring.

This is a highly interesting contribution for the detailed understanding of excitation energy transport in photosynthetic complexes, which is a rather complex process involving the interplay of electronic coupling, vibronic coupling, and the environment.

The paper is clearly written, and the conclusions are well supported by the experimental results. I recommend its acceptance after the authors address the following minor points

1. Is the ring symmetry largely preserved during the exciton relaxation? how large is the amplitude of the nuclear motion that breaks the symmetry? Is this something that can be determined from the 2DES?

2. Regarding the vibrational coherences generated during the exciton relaxation, is it possible to use e.g. a Raman or infrared technique to probe, which could provide a more conclusive evidence for their roles in the exciton dynamics? A discussion of this will be useful. 
Reviewer: 2

Comments to the Author

Manuscript ID: jz-2021-038682

Title: "Vibrational Modes Promoting Exciton Relaxation in the B850 Band of LH2"

Authors: Kim, JunWoo; Nguyen-Phan, Tu; Gardiner, Alastair; Yoon, Tai Hyun; Cogdell, Richard; Cho, Minhaeng; Scholes, Gregory

The work under review focuses on the experimental identification of the vibrational modes responsible for the ultrafast relaxation of the $k= \pm 1$ excitons in the B850 light-harvesting complex from Rhodoblastus acidophilus using polarization-dependent transient two-dimensional electronic spectroscopy. The method relies on an expected $\sim 90$-degree change of the transition dipole angle between the quasi-degenerate $k= \pm 1$ exciton states upon the energy transfer. A vibrational coherence redistribution from the high-frequency modes of the donor state to the low-frequency modes of the acceptor state was found, revealing the modes that most effectively facilitate the exciton relaxation.

While the obtained results appear interesting, several questions require clarification.

Experimental data are provided for just one combination of experimental parameters: $\lambda e x=860 \mathrm{~nm}$, $\lambda \mathrm{re}=870 \mathrm{~nm}, \mathrm{~T}=0.5 \mathrm{ps}$. I wonder, how does the data change upon varying these parameters? Especially the dependence on $\mathrm{T}$ is relevant to support the conclusions, I suppose.

The observed oscillations have been related to the excited-state vibrational wavepackets. How the possible interference with the ground-state vibrational wavepackets have been avoided/removed, considering the broadband fs-pulse excitation inevitably simultaneously generates both ground-state and excited-state vibrational wavepackets?

What is the noise/uncertainty in the FT power spectrum of Fig. 3b?

I am also missing an interpretation of the low-frequency vibrations (including the 289- $\mathrm{cm}-1$ mode) that overlap with the quasi-continuous phonon spectrum of $\mathrm{LH} 2$.

The experimental data are analyzed by a simplified model using several approximations such as (i) the vibronic coupling and the exciton relaxation dynamics are identical for the two exciton states (p. 7); (ii) the oscillatory strengths of the $k= \pm 1$ transitions are identical (p. 9); (iii) the splitting of the $k= \pm 1$ is due solely to geometrical distortion of the B850 (p. 9). As we well know, none of these approximations hold in the real (statically and dynamically disordered) world. Therefore, an analysis is in order to explain how the applied model approximations may modify the interpretation of the data.

In conclusion: Although the presented manuscript contains interesting data, it is not publishable at this stage. Additional data analyses are required to properly support all the conclusions made.

Reviewer: 3 
Comments to the Author

Reviewer response to "Vibrational modes promoting exciton relaxation in the B850 band of LH2" by Kim et al:

This paper builds on a previous work by some of the authors (ref 44) of utilizing 2DES by a pair of synchronized mode-locked laser toward analyzing the vibronic aspects of exciton relaxation involved with the interconversion between two bright states of LH2 B850 band. Although LH2 has been heavily studied with diverse techniques, its complete understanding is far from being reached yet. The present work definitely delivers new information that is peculiar in that it covers a very wide time range thanks to the technical development in ref 44 and in that many dynamically important vibrational peaks are well resolved.

The above advancements are indeed significant in that such efforts have long been sought for in the community, and the present work will play a role of promoting discussions by both experimentalists and theoreticians who study the physical chemistry of photosynthesis and other related exciton transfer/conversion processes. Thus, I feel the paper will find many interests from the readers of JPCL.

Before its publication, however, I wish the authors to clarify on the following aspects.

1. The ultrafast exciton relaxation (more than 0.5 within $20 \mathrm{fs}$, Figure S1) is interesting. In fact, considering the energy gap between the $k=+1 /-1$ states, $\sim 0.6$ is a complete transfer allowed by the Boltzmann factor. (Noting this on the paper will likely be a good idea.) However, I am a bit confused by the way of interpreting the role of vibrations that are decreasing in their intensities from $D<--D$ to $A<--D$ in Figure $4 \mathrm{~b}(30,90,289,347,567 \mathrm{~cm}-1)$, especially the high frequency ones $(289,347$, and $567 \mathrm{~cm}-1)$.

1-1. Doesn't the fact that these three are strong with $D<--D$ but almost non-existing with $A<--D$ mean that they are strongly coupling for $D<--G$ transition but not for $A<--D$ transition (exciton relaxation)?

1-2. Figure 4 suggests that the fast symmetry-breaking modes (two of the three) induce the exciton relaxation by "driving the system to a geometry where the two exciton states coupled to each other". However, the vibrational periods of the two modes at 347 and $567 \mathrm{~cm}^{\wedge}-1$ are 96 and $59 \mathrm{fs}$, and are quite slower than exciton relaxation time of $\sim 20 \mathrm{fs}$ of the major decay path. I am not sure whether they are "driving actively on" or "driven impulsively by" electronic state conversion.

2. The modes below $100 \mathrm{~cm}-1$. They are is in a region where a soup of phonon modes tend to exist. Therefore, care must be exercised on distinguishing which is which. The authors explain that the fast symmetry-breaking modes pass kinetic energy to these modes. Can they discuss the nature of the low frequency modes somewhat more? Energy exchanges between modes with widely different frequencies 
tend to take quite some time unless they are tied through chemical bonds ( $\sim 10 \mathrm{ps}$ or even longer in my experience), and I am curious about what exactly is going on. Being at low frequency may mean that they possess strong intermolecular nature, which is devoid of direct chemical bonds. If this is the case, what is the reason the vibrational conversion is fast? I understand nailing down what it is is not an easy task, but at least some guessing might be done here than just reading the peaks.

3. In the same context, what will be the nature of $289 \mathrm{~cm}-1$ mode? I am pretty sure it will be an intramolecular mode as it is at high enough frequency, but the authors say that it does not couple to single chromophore transition. Does this mean that a mode somehow becomes active through forming some sort of delocalization over multiple chromophores (a collective mode)?

4. Minor stuffs:

4-1. Just before eq 2, it would help to show the starting definition of rho_A. Something like:

"Therefore, the relative (normalized) population of the A-state created by the exciton relaxation from $D$ to A, I_SE,A-D/I_GSB, can be written as"

4-2. The horizontally wrinkling structure of rho in Figure $4 \mathrm{~b}$ is the most important data. Can the authors state how large the excitation wavelength dependence was? (up to the limit of not getting affected by the relaxations from higher exciton states than $+/-1$ )

4-3. Please revise the last sentence just before Notes.

Author's Response to Peer Review Comments: 


\section{Reviewer: 1}

The authors carried out a comprehensive experimental study of the exciton relaxation in the B850 band of LH2 using two-dimensional electronic spectroscopy. By analyzing the spectra, the authors identified several vibrational modes coupled to the electronic transitions that can play an important role in the exciton relaxation, especially the modes that breaks the symmetry of the ring.

This is a highly interesting contribution for the detailed understanding of excitation energy transport in photosynthetic complexes, which is a rather complex process involving the interplay of electronic coupling, vibronic coupling, and the environment.

The paper is clearly written, and the conclusions are well supported by the experimental results. I recommend its acceptance after the authors address the following minor points

Comment 1. Is the ring symmetry largely preserved during the exciton relaxation? how large is the amplitude of the nuclear motion that breaks the symmetry? Is this something that can be determined from the $2 \mathrm{DES}$ ?

Reply 1. Possible ring symmetry-breaking modes were not clearly identified in this study. We hypothesize that they are implicated in the mixing of the $k= \pm 1$ exciton states, much like strain mixes exciton states of total angular momentum $k= \pm 1$ in semiconductors. See ref 34 [J. Phys. Chem. C 2009, 113 (3), 795-811]. We have added a sentence in the third paragraph on page 12 to clarify what we observed.

"Similarly, a ring symmetry-breaking mode may affect the exciton relaxation dynamics. Though we could not identify such acoustic phonon modes, we hypothesize that they are implicated in the mixing of the $k= \pm 1$ exciton states, much like strain mixes exciton states of total angular momentum $k= \pm 1$ in semiconductors ${ }^{34}$." 
Comment 2. Regarding the vibrational coherences generated during the exciton relaxation, is it possible to use e.g. a Raman or infrared technique to probe, which could provide a more conclusive evidence for their roles in the exciton dynamics? A discussion of this will be useful.

Reply 2. We fully agree with the reviewer 1's opinion. To show the role of vibrational motion for the exciton relaxation, the correlation among the vibrational energy donor $\left(289,347\right.$, and $567 \mathrm{~cm}^{-1}$ modes), acceptor (30 and $90 \mathrm{~cm}^{-1}$ modes), and the associated electronic $(k= \pm 1)$ transitions needs to be identified simultaneously. Time-resolved Raman spectroscopy (e.g., femtosecond stimulated Raman spectroscopy and time-resolved impulsive stimulated Raman spectroscopy) and transient 2DIR spectroscopy have a capability of measuring such correlation. Accordingly, we have added a short discussion at the last paragraph.

"The correlation between the high- and low-frequency modes with respect to the exciton relaxation can be clarified using fifth-order spectroscopy techniques such as time-resolved Raman ${ }^{45}$ spectroscopy." 


\section{Reviewer: 2}

The work under review focuses on the experimental identification of the vibrational modes responsible for the ultrafast relaxation of the $\mathrm{k}= \pm 1$ excitons in the B850 light-harvesting complex from Rhodoblastus acidophilus using polarization-dependent transient two-dimensional electronic spectroscopy. The method relies on an expected $\sim 90$-degree change of the transition dipole angle between the quasi-degenerate $\mathrm{k}= \pm 1$ exciton states upon the energy transfer. A vibrational coherence redistribution from the high-frequency modes of the donor state to the low-frequency modes of the acceptor state was found, revealing the modes that most effectively facilitate the exciton relaxation.

While the obtained results appear interesting, several questions require clarification.

Comment 1. Experimental data are provided for just one combination of experimental parameters: $\lambda$ ex $=860 \mathrm{~nm}, \lambda \mathrm{re}=870 \mathrm{~nm}, \mathrm{~T}=0.5 \mathrm{ps}$. I wonder, how does the data change upon varying these parameters? Especially the dependence on T is relevant to support the conclusions, I suppose.

Reply 1. We thank the reviewer 2 for pointing out the observation window. As the reviewer pointed out, we should have shown (1) the effect of spectral evolution over $T$ and (2) the optical frequency dependence on the wavepacket analysis.

(1) To show the $2 \mathrm{D}$ spectral evolution over $T$, we have added the $2 \mathrm{D}$ electronic spectra at several $T$ points (100 fs, $500 \mathrm{fs}, 1 \mathrm{ps}$, and 5 ps) in the revised Supporting information (Figure S3). Also, we added a sentence in the last paragraph on page 8 to mention the negligible contribution of the spectral evolution as

"We checked that the spectral evolutions of the 2D electronic spectra are sufficiently negligible (Figure S3) within the Fourier transformation window (120 fs $-4.5 \mathrm{ps}$ ) for the wavepacket analysis." 
(2) To show the optical frequency dependence on the wavepacket analysis, we added the 2D $\left(\lambda_{\text {exc }} \times\right.$

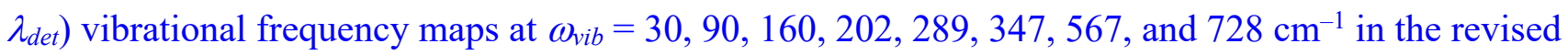
Supporting information (Figure S4).

"The wavepacket oscillations belonging to the ESA may interfere the wavepacket analysis because it does not provide selectivity for the $D$ - and $A$-states as explained above, and because the vibronic coupling structures of the B850 ESA and the B850 absorption are obviously different to each other (Figure S4).”

Comment 2. The observed oscillations have been related to the excited-state vibrational wavepackets. How the possible interference with the ground-state vibrational wavepackets have been avoided/removed, considering the broadband fs-pulse excitation inevitably simultaneously generates both ground-state and excited-state vibrational wavepackets?

Reply 2. As the reviewer 2 commented, distinguishing the vibrational wavepackets generated on the ground state and an excited state is an important issue for excited-state dynamics studies based on wavepacket analysis. The wavepackets cannot be clearly separated unless stimulated emission and ground-state bleach bands are separated in transition energy. Therefore, the wavepackets shown in the main text should have both the excited- and ground-state contributions.

We omitted the discussions on the ground-state wavepackets in the original manuscript due to the negligible contribution of the ground-state response to the exciton relaxation. The vibrational coherence on the ground state is not perturbed by any electronic-state conversion like the ultrafast exciton relaxation. Therefore, the polarization dependence shown in Figure 3 is mostly attributed to the excited state (except the random orientation effect), so that we do not discuss about the groundstate wavepacket in this study. 
For better understanding of readers, we have added a brief discussion on the ground-state wavepacket at the first paragraph on page 11.

"The wavepackets in Figure 3 are the mixture of the wavepackets on the ground and on the $k=$ \pm 1 states. The contribution of the ground-state response needs to be excluded for an excited-state dynamics study. In this study, the vibrational coherence on the ground state is not perturbed by the ultrafast exciton relaxation. Therefore, the polarization dependence shown in Figure 3 is mostly attributed to the excited state, so that we do not discuss about the ground-state wavepacket in this study."

Comment 3. What is the noise/uncertainty in the FT power spectrum of Fig. 3b?

Reply 3. The accuracy of the peak positions in $\tilde{I}_{\mathrm{D}-\mathrm{D}}(\omega)$ and $\tilde{I}_{\mathrm{A}-\mathrm{D}}(\omega)$ are determined by the data quality of raw data (the Fourier transforms of $I_{\text {para,osc }}(T), \tilde{I}_{\text {para }}(\omega)$, and $\left.I_{\text {perp,osc }}(T), \tilde{I}_{\text {perp }}(\omega)\right)$ because $\tilde{I}_{\mathrm{D}-\mathrm{D}}(\omega)$ and $\tilde{I}_{\mathrm{A}-\mathrm{D}}(\omega)$ are the linear combinations of the raw data. For $\tilde{I}_{\mathrm{para}}(\omega)$ and $\tilde{I}_{\text {perp }}(\omega)$, the uncertainties $\left(\tilde{I}_{\text {background }}\right.$ / $\left.\tilde{I}_{\text {peak }}\left(\omega=728 \mathrm{~cm}^{-1}\right)\right)$ of the weakest vibrational coherence peak $\left(728 \mathrm{~cm}^{-1}\right)$ are $1 / 9.5$ and $1 / 3.8$, respectively. Here, $\tilde{I}_{\text {background }}$ is defined as the mean noise-level multiplied by 3 to estimate the peak amplitude with 3-sigma confidence. To confirm our data quality, we added the coherent vibrational spectra (the Fourier transforms of $I_{\text {para,osc }}(T)$ and $I_{\text {perp,osc }}(T)$ ) of the raw data in Supporting information (Figure S5 in the revised SI).

$\tilde{I}_{\mathrm{D}-\mathrm{D}}(\omega)$ and $\tilde{I}_{\mathrm{A}-\mathrm{D}}(\omega)$ look more noisy than raw data, where the uncertainties at $728 \mathrm{~cm}^{-1}$ are $1 / 7.5$ and $1 / 4.2$, respectively. These could be caused by random constructive interference between the background noises of $I_{\text {para,osc }}(T)$ and $I_{\text {perp,osc }}(T)$. However, the signal-to-noise ratios (uncertainties) of them are still high (low) enough to compare the coherent vibrational peaks in $\tilde{I}_{\mathrm{D}-\mathrm{D}}(\omega)$ and $\tilde{I}_{\mathrm{A}-\mathrm{D}}(\omega)$ since the peak position is well estimated by the higher data quality of the raw data. 


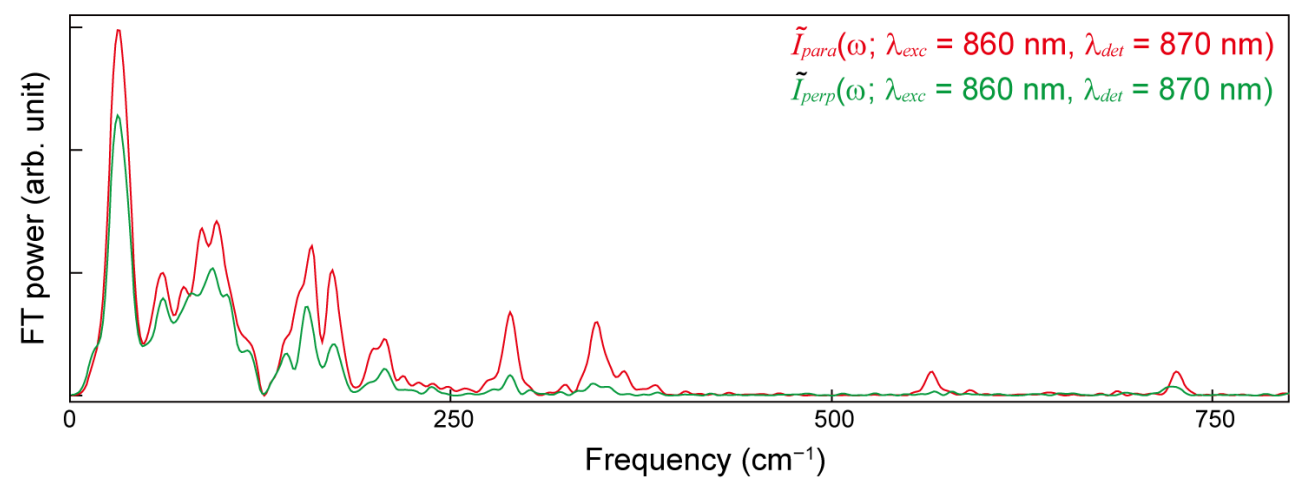

Figure S5. Coherent vibrational spectra of LH2 measured at ( $\lambda_{\text {exc }}=860 \mathrm{~nm}, \lambda_{\text {det }}=870 \mathrm{~nm}$ ) with the parallel $\left(\tilde{I}_{\text {para }}(\omega)\right.$, red $)$ and perpendicular $\left(\tilde{I}_{\text {para }}(\omega)\right.$, green $)$ probes. The signal-to-noise ratio of $\tilde{I}_{\text {para }}(\omega)$ and $\tilde{I}_{p e r p}(\omega)$ are 9.5 and 3.8 , respectively, at $728 \mathrm{~cm}^{-1}$, where the noise level is the averaged background level multiplied by 3 .

Comment 4. I am also missing an interpretation of the low-frequency vibrations (including the 289cm-1 mode) that overlap with the quasi-continuous phonon spectrum of LH2.

Reply 4. The quasi-continuous phonon spectrum should appear as overdamped oscillation. The contribution of them has been removed since we extracted oscillating trajectories only. The quasicontinuous phonon may play a role for the ultrafast exciton relaxation. Unfortunately, however, the contribution of quasi-continuous phonon modes for an interexciton dynamics is indistinguishable from typical photoinitiated reorganization process caused by environmental reorientation (e.g., solvation) in 2DES study.

Comment 5. The experimental data are analyzed by a simplified model using several approximations such as (i) the vibronic coupling and the exciton relaxation dynamics are identical for the two exciton states (p. 7); (ii) the oscillatory strengths of the $\mathrm{k}= \pm 1$ transitions are identical (p. 9); (iii) the splitting of the $\mathrm{k}= \pm 1$ is due solely to geometrical distortion of the B850 (p. 9). As we well know, none of these approximations hold in the real (statically and dynamically disordered) world. Therefore, an analysis is in order to explain how the applied model approximations may modify the interpretation of the data. 
Reply 5. We thank the reviewer 3 for the critical comment about our assumptions. As the reviewer pointed out, the vibronic coupling structures and the oscillator strengths of the $k= \pm 1$ states cannot be identical. We guess our description for the data analysis was not good enough for the readers.

What we assume is the identical vibronic coupling structures and the oscillators strengths of the donor $(D)$ and acceptor $(A)$ states, not those properties of the $k= \pm 1$ states. In this study, the $D$ - and $A$ states are defined as the photoexcited state and exciton transferred state, respectively, so that both the $D$ - and $A$-states are the statistically averaged exciton states of the $k= \pm 1$ states in the experimental point of view. Because (1) we have chosen the excitation wavelength as where the $k= \pm 1$ absorption bands well overlap $\left(\lambda_{\text {exc }}=860 \mathrm{~nm}\right)$ and (2) the $k= \pm 1$ states have similar electronic structure due to their quasi-degeneracy, the $D$ - and $A$-states should show almost identical vibronic coupling structure and oscillator strengths. Therefore, the polarization dependence in the wavepacket oscillation of the B850 ring should be attributed to the vibrational coherence reorganization accompanied by the ultrafast relaxation.

Lastly, it should be noted that the relative vibrational peak amplitudes in $\tilde{I}_{p a r a}(\omega)$ and in $\tilde{I}_{p e r p}(\omega)$ (wavepackets measured with parallel and perpendicular probes) are not identical (please see Figure S5 attached in Reply 3). This means that the polarization dependence is not solely due to the random orientation (isotropic ensemble measurement) effect. To remove the contribution of the random orientation, we carried out an additional data analysis described in equation 2 and Supporting information section 1.

We have rewritten the sentences associated with the comments $i$ and ii. According to the comment $i$, the sentence in the third paragraph on page 7 was modified from

"Since the oscillator strengths of the $k= \pm 1$ transitions are identical, ..." 
"Since the oscillator strengths of the $D$ - and $A$-transitions, which are the statistical averaged states of the $k= \pm 1$ transitions, are almost the same, ..."

For the comment ii, the sentences in the last paragraph on page 5 are modified from

"The $k= \pm 1$ states should have the same electronic characteristics, consistent with their energy degeneracy. Therefore, we assume that the vibronic coupling and the exciton relaxation dynamics are identical for the two exciton states. In this article, an initially excited state, either the $k=+1$ or the $k=-1$ state, is defined as the exciton donor $(D)$ state, and thus the other state is assigned to the exciton acceptor $(A)$ state."

to

"Because the $k= \pm 1$ states are experimentally indistinguishable, an initially excited state, either the $k=+1$ or the $k=-1$ state, is defined as the exciton donor $(D)$ state, and thus the other state is assigned to the exciton acceptor $(A)$ state to explain the polarization dependence associated with the exciton relaxation. This implies that the $D$ - and $A$-states are the statistically averaged states of the $k= \pm 1$ states. Therefore, we can assume that the vibronic coupling structures of the $D$ - and $A$-states are identical, and the polarization dependence in the vibrational wavepacket of the $\mathrm{B} 850$ ring should be attributed to the exciton relaxation $(\mathrm{D} \rightarrow \mathrm{A}$ transition)."

For the comment iii, we have revised the corresponding sentence (the last paragraph on page 8 in the revised manuscript) according to the comment from

"This excitation wavelength dependence should be attributed to the small ellipticity of the B850 ring which lifts the degeneracy, giving an $\sim 110 \mathrm{~cm}^{-1}$ energy difference between the $k= \pm 1$ states (Figure 1b)."

to

"This excitation wavelength dependence should be attributed to the small ellipticity and disorder of the B850 ring which lifts the degeneracy, giving an $\sim 110 \mathrm{~cm}^{-1}$ energy difference between the $k= \pm 1$ states (Figure 1b).”. 


\section{Reviewer: 3}

This paper builds on a previous work by some of the authors (ref 44) of utilizing 2DES by a pair of synchronized mode-locked laser toward analyzing the vibronic aspects of exciton relaxation involved with the interconversion between two bright states of LH2 B850 band. Although LH2 has been heavily studied with diverse techniques, its complete understanding is far from being reached yet. The present work definitely delivers new information that is peculiar in that it covers a very wide time range thanks to the technical development in ref 44 and in that many dynamically important vibrational peaks are well resolved.

The above advancements are indeed significant in that such efforts have long been sought for in the community, and the present work will play a role of promoting discussions by both experimentalists and theoreticians who study the physical chemistry of photosynthesis and other related exciton transfer/conversion processes. Thus, I feel the paper will find many interests from the readers of JPCL.

Before its publication, however, I wish the authors to clarify on the following aspects.

Comment 1. The ultrafast exciton relaxation (more than 0.5 within $20 \mathrm{fs}$, Figure S1) is interesting. In fact, considering the energy gap between the $\mathrm{k}=+1 /-1$ states, $\sim 0.6$ is a complete transfer allowed by the Boltzmann factor. (Noting this on the paper will likely be a good idea.) However, I am a bit confused by the way of interpreting the role of vibrations that are decreasing in their intensities from $\mathrm{D}<-$-D to $\mathrm{A}<-$-D in Figure 4b $(30,90,289,347,567 \mathrm{~cm}-1)$, especially the high frequency ones (289, 347 , and $567 \mathrm{~cm}-1)$.

Comment 1-1. Doesn't the fact that these three are strong with $\mathrm{D}<-$-D but almost non-existing with $\mathrm{A}<-$-D mean that they are strongly coupling for $\mathrm{D}<-$-G transition but not for $\mathrm{A}<-$-D transition (exciton relaxation)? 
Reply 1-1. If the coupling strength of a vibrational mode is weak with respect to the $\mathrm{A} \leftarrow \mathrm{D}$ transition, the potential energy surface of the mode will be similar in the $k=+/-1$ excited states. This implies that the photoinitiated vibrational coherence of the mode should be preserved after the exciton relaxation.

When the vibronic coupling strength of a mode for the $A \leftarrow D$ transition is much stronger than the $\mathrm{D} \leftarrow \mathrm{G}$ transition, the coherence of the mode can be generated by the exciton relaxation as the reviewer expected. In the B850 ring case, however, the vibronic coupling for the $\mathrm{A} \leftarrow \mathrm{D}$ transition could be much weaker than the $\mathrm{D} \leftarrow \mathrm{G}$ transition because (1) both the $k= \pm 1$ states can be expressed as the linear combination of monomeric electronic states, and because (2) the reactive $(289,347$, and 567 $\mathrm{cm}^{-1}$ ) modes have high monomeric characters.

Comment 1-2. Figure 4 suggests that the fast symmetry-breaking modes (two of the three) induce the exciton relaxation by "driving the system to a geometry where the two exciton states coupled to each other". However, the vibrational periods of the two modes at 347 and $567 \mathrm{~cm}^{\wedge}-1$ are 96 and $59 \mathrm{fs}$, and are quite slower than exciton relaxation time of $\sim 20 \mathrm{fs}$ of the major decay path. I am not sure whether they are "driving actively on" or "driven impulsively by" electronic state conversion.

Reply 1-2. The symmetry-breaking coordinate in Figure 4 can be defined as a linear combination of the three $\left(289,347\right.$, and $\left.567 \mathrm{~cm}^{-1}\right)$ vibrational modes. The exciton relaxation would take place when a wavepacket passes a specific state-of-motion (position and momentum) on the symmetry-breaking coordinate, where the interexciton coupling is sufficiently strong. The probability for the exciton relaxation rapidly increases as the wavepacket gets close to the effective state-of-motion. Therefore, the exciton-relaxation time can be shorter or longer than the half-period of a relevant vibrational mode, where the half-periods of the three modes $(58,48$, and $30 \mathrm{fs})$ are quite comparable to the relaxation time. The ultrafast excited-state intramolecular proton transfer (18 fs) of 10-hydroxybenzo[ $h]$ quinoline (HBQ) [J. Phys. Chem. Lett. 2020, 11 (3), 755-761] and the ultrafast exciton relaxation (<100 fs) in 
semiconductor nanocrystals [J. Phys. Chem. C 2009, 113 (3), 795-811] are appropriate examples, where the half periods of associated vibrational and phonon modes are 65 fs and 5.5 fs for HBQ, and $>3$ ps for nanocrystals.

For better understanding of readers, we have revised the description for the Figure 4 (the third paragraph on page 13).

"As the photoexcited B850 ring approaches the geometry, the probability for the exciton relaxation dramatically increases. Then, the exciton relaxation occurs even before the half period of any reactive vibration. Finally, the vibrational kinetic energy of the symmetry-breaking modes is transferred to the low-frequency modes of the $A$-state."

Comment 2. The modes below $100 \mathrm{~cm}-1$. They are is in a region where a soup of phonon modes tend to exist. Therefore, care must be exercised on distinguishing which is which. The authors explain that the fast symmetry-breaking modes pass kinetic energy to these modes. Can they discuss the nature of the low frequency modes somewhat more? Energy exchanges between modes with widely different frequencies tend to take quite some time unless they are tied through chemical bonds $(\sim 10$ ps or even longer in my experience), and I am curious about what exactly is going on. Being at low frequency may mean that they possess strong intermolecular nature, which is devoid of direct chemical bonds. If this is the case, what is the reason the vibrational conversion is fast? I understand nailing down what it is not an easy task, but at least some guessing might be done here than just reading the peaks.

Reply 2. We thank the reviewer 3 for the critical comment on vibrational energy transfer dynamics during the exciton relaxation. As explained in the manuscript, the low-frequency $\left(<100 \mathrm{~cm}^{-1}\right)$ modes are the vibrational modes involved in the vibronic coupling between the $k= \pm 1$ states. Unfortunately, we cannot discuss more about these modes since we don't know the nuclear motion information of them. 
It has been reported that a $30 \mathrm{~cm}^{-1}$ mode shows a strong vibronic coupling with respect to the $Q_{y}$-transition of free-base porphyrin molecule, which is a structural analogue of the monomeric unit of the B850 ring (BChl a) [J. Phys. Chem. Lett. 2015, 6, 2993-2998]. Therefore, we expect that the 30 $\mathrm{cm}^{-1}$ mode has a high monomeric character. Intermolecular vibrations may have lower $\left(<10 \mathrm{~cm}^{-1}\right)$ frequency because $\mathrm{BChl} a$ is a super heavy $(\mathrm{mw}=911.50 \mathrm{~g} / \mathrm{mol})$ molecule.

As the reviewer pointed out, intramolecular vibrational redistribution of non-reactive chromophores typically takes place in picosecond time window through the anharmonic coupling. However, if a photoexcited chromophore undergoes an instantaneous electronic-state conversion (20fs exciton relaxation in this study), the wavepacket motion generated by photoexcitation is converted (redistributed) to the vibrational motions of the final electronic state.

Comment 3. In the same context, what will be the nature of $289 \mathrm{~cm}-1$ mode? I am pretty sure it will be an intramolecular mode as it is at high enough frequency, but the authors say that it does not couple to single chromophore transition. Does this mean that a mode somehow becomes active through forming some sort of delocalization over multiple chromophores (a collective mode)?

Reply 3. We fully agree with the reviewer 3's opinion that the $289 \mathrm{~cm}^{-1}$ mode originates from single molecular vibration. There seems to have been a confusion due to our poor description. What we tried to say is that this mode does not show high vibronic coupling strength with respect to the $Q_{y}$ transition of BChl $a$. The strong $\left(\sim 300 \mathrm{~cm}^{-1}\right)$ electronic coupling among the monomeric units (BChl $a$ s) could cause the vibronic coupling of the mode. We have rewritten the corresponding sentence (from the first paragraph on page 13) to avoid confusion.

"The $289 \mathrm{~cm}^{-1}$ mode could be a single molecular vibrational mode according to its high enough frequency. However, we could not assign the $289 \mathrm{~cm}^{-1}$ mode because the mode does not show significant vibronic coupling strength in a single pigment BChl $a . "$ 
Comment 4. Just before eq 2, it would help to show the starting definition of rho_A. Something like: "Therefore, the relative (normalized) population of the A-state created by the exciton relaxation from D to A, I_SE,A-D/I_GSB, can be written as"

Reply 4. Thanks for a helpful comment. We have rewritten the sentence as the reviewer suggested.

Comment 5. The horizontally wrinkling structure of rho in Figure $4 \mathrm{~b}$ is the most important data. Can the authors state how large the excitation wavelength dependence was? (up to the limit of not getting affected by the relaxations from higher exciton states than $+/-1$ )

Reply 5. We appreciate to the reviewer 3 for the comment about excitation wavelength $\left(\lambda_{\text {exc }}\right)$ dependence on $\rho_{A}(T)$. We added the $\lambda_{\text {exc }}$-dependent acceptor population, $\rho_{A}\left(\lambda_{e x c} ; T=1 \mathrm{ps}\right)$, in the revised Supporting information (Figure S3) to estimate the contribution from higher $|k|$ states. Finally, we found that $\rho_{A}\left(\lambda_{e x c} ; T=1 \mathrm{ps}\right)$ is slightly higher around $\lambda_{e x c}=825 \mathrm{~nm}$. Theoretical model [Chem. Rev. 2017, 117 (2), 249-293] suggests that the energy levels of the $k= \pm 2$ states are $300 \mathrm{~cm}^{-1}$ higher than the $k= \pm 1$ states. Therefore, the $\lambda_{\text {exc }}$-dependence around $\lambda_{\text {exc }}=825 \mathrm{~nm}$ should be attributed to the blueedge excitation of the $k= \pm 2$ states.

There is a rapid decrease in $\rho_{A}(T=1 \mathrm{ps})$ from $\lambda_{\text {exc }}=825 \mathrm{~nm}$ to $\lambda_{\text {exc }}=835 \mathrm{~nm}$. This represents the negligible contribution of the $k= \pm 2$ states inside the $k= \pm 1$ absorption lines due to dramatic oscillator strength difference between the $k= \pm 1$ and $k= \pm 2$ absorptions.

Accordingly, we mentioned Figure S3 in the last paragraph on page 8, in which the negligible contribution of the exciton relaxation from $k= \pm 2$ states is discussed. 
Comment 6. Please revise the last sentence just before Notes.

Reply 6. Thanks for pointing out that. We have rewritten the last sentence as

"Interestingly, the vibrational modes that break the pseudo- $D_{4 \mathrm{~h}}$ symmetry of the monomoric units are more strongly correlated with the exciton relaxation than the symmetric vibrations. It is known that symmetry-breaking modes can modulate the electronic property of excited monomer (BChl $a$ ), for example they mix the $Q_{y}$ and $Q_{x}$ states $^{45}$. The periodic and monomeric electronic property modulations can collectively (weakly) perturb the exciton structure in time, and therefore allow exciton relaxation." 
Name: Peer Review Information for "Vibrational Modes Promoting Exciton Relaxation in the B850 Band of LH2"

Second Round of Reviewer Comments

Reviewer: 2

Comments to the Author

I appreciate the explanations provided by the authors and the amendments made in the revised manuscript. Although not conclusively, the applied polarization-dependent transient two-dimensional electronic spectroscopy approach allows revealing new aspects of exciton relaxation in the LH2 photosynthetic antenna complexes.

Reviewer: 3

Comments to the Author

The authors have addressed my comments properly, and I am now fully supportive about their publication. Two points they may consider (optionally):

1. The introduction about the newly added Fig $4 \mathrm{~S}$ is too abrupt in the main text. How about putting the comment in the response letter to the main text itself? ("To show the optical frequency dependence on the wavepacket analysis, we [also present] the 2D vibrational frequency maps at wvib $=30,90,160$, $202,289,347,567$, and $728 \mathrm{~cm}-1$ in

Figure S4."

2. One paragraph in the response letter, "When the vibronic coupling strength of a mode for the $A \leftarrow D$ transition is much stronger than the $D \leftarrow G$ transition, the coherence of the mode can be generated by the exciton relaxation as the reviewer expected. In the B850 ring case, however, the vibronic coupling for the $A \leftarrow D$ transition could be much weaker than the $D \leftarrow G$ transition because (1) both the $k= \pm 1$ states can be expressed as the linear combination of monomeric electronic states, and because (2) the reactive $(289,347$, and $567 \mathrm{~cm}-1)$ modes have high monomeric characters." 
A short description similar to this in the main text will be helpful for the readers too, unless there is a pressure from a length limit dictated by JPCL.

Author's Response to Peer Review Comments:

Thank you, we have made these revisions suggested by the reviewers, as well as a the editorial changes Greg 